\title{
Does inflammation get on nerves in patients with heart failure?
}

\author{
Albert Flotats, $M D,^{a, b, c}$ and Ignasi Carrio, $M D^{a, b, c}$ \\ a Nuclear Medicine, Hospital de la Santa Creu i Sant Pau, Barcelona, Catalunya, Spain \\ b Universitat Autonoma de Barcelona, Bellaterra, Catalunya, Spain \\ c Sant Pau Biomedical Research Institute (IIB Sant Pau), Barcelona, Catalunya, Spain
}

Received Nov 9, 2016; accepted Nov 9, 2016

doi: $10.1007 / \mathrm{s} 12350-016-0754-2$

\section{See related article, pp. 845-853}

Heart failure (HF) is a major public health burden in developed countries, with elevated morbidity and mortality upon diagnosis. Therefore, effective treatment and prognosis are critical in the management of HF.

Pathophysiologic changes induced by myocardial injury activate the sympathetic system, which initially helps to compensate for impaired left ventricular (LV) function. However, persistent activation of this system in chronic HF has detrimental consequences including arterial and venous constriction by release of vasoactive substances, activation of renin-angiotensin-aldosterone system (RAAS) with salt and water retention and increased myocardial wall stress and oxygen and energy requirements, along with down regulation of postsynaptic $\beta$-adrenoreceptors, culminating in ventricular remodeling and myocardial fibrosis.

There are many reports showing that as clinical status and/or LV function improve in response to HF medical treatment, there is a corresponding improvement in cardiac sympathetic nerve function as assessed by Iodine123 meta-iodobenzylguanidine $\left({ }^{123} \mathrm{I}-\mathrm{mIBG}\right)$, supporting that cardiac sympathetic imaging is important for risk stratification in HF patients. ${ }^{1,2}$ AdreView Myocardial Imaging for Risk Evaluation in Heart Failure (ADMIRE$\mathrm{HF}$ ), up-to-date the largest prospective multicenter study, validated the prognostic assessment of cardiac ${ }^{123} \mathrm{I}-\mathrm{mIBG}$ imaging in patients with HF. The study included $961 \mathrm{HF}$

Reprint requests: Albert Flotats, MD, Nuclear Medicine, Hospital de la Santa Creu i Sant Pau, Barcelona, Catalunya, Spain; aflotats@ santpau.cat

J Nucl Cardiol 2018;25:854-6.

1071-3581/\$34.00

Copyright (C) 2016 American Society of Nuclear Cardiology. patients in New York Heart Association (NYHA) functional class II-III with ischemic or non-ischemic cardiomyopathy with a site-reported LV ejection fraction $(\mathrm{LVEF}) \leq 35 \%$, who were on guideline-recommended medical therapy and followed-up for a median of 17 months. Late ${ }^{123}$ I-mIBG heart-to-mediastinum (H/R) ratio demonstrated its value in predicting the primary composite endpoint of cardiac death, potentially lifethreatening arrhythmias, or HF progression. ${ }^{3}$ Preserved $\mathrm{H} / \mathrm{M}$ ratio showed a high negative predictive value with respect to cardiac death or cumulative arrhythmic events, which confirmed results from the previous studies in less consistent populations. In addition, H/M ratio demonstrated an independent prognostic capability complementary to other commonly used markers such as LVEF and B-type natriuretic peptide (BNP), and may help differentiate patients likely to benefit from medical therapy from those that are likely to show poor long-term response and thus might better benefit from other treatments such as device therapy and cardiac transplantation.

In parallel, there is growing evidence supporting the hypothesis that inflammation plays a key role in the progression and worsening of $\mathrm{HF}^{4}$ Myocardial injury would induce an inflammatory response to resolve the source of the disturbance, thereby allowing the heart to adapt to the abnormal conditions in the short term, and finally to restore homeostasis and cardiovascular function in the long term. However, if the abnormal circumstances are sustained, a persistent inflammatory state would continue in the tissue and would lead to a state of chronic low-grade inflammation, which could contribute to further disease progression due to the deleterious effects of sustained inflammation. Therefore, analogous to sustained neurohormonal activation in $\mathrm{HF}$, the chronic inflammation that occurs in HF may also contribute to worsening the ventricular function due to the harmful effects of sustained inflammatory signaling. Of note, activation of neurohormonal systems in HF, such as the sympathetic nervous 
system and the RAAS, could trigger inflammation in the heart, thus leading to a state of low-grade inflammation. ${ }^{4}$

Actually, elevated serum levels of inflammatory mediators have been identified in patients with HF, and experimental studies have shown that activation of inflammation in the heart produces LV remodeling and LV dysfunction. In addition to their direct involvement in the pathogenesis of $\mathrm{HF}$, inflammatory mediators could also be the suitable markers for risk stratification and prognostication in patients with HF. Many reports have suggested that inflammatory mediators may predict adverse outcome in these patients. However, most studies have been limited in sample size and lacking full adjustment with the most recent and strongest biochemical predictor such as NT-proBNP and highsensitivity troponins. Furthermore, a number of pre-analytical and analytical aspects of inflammatory mediator measurements may limit their use as biomarkers. Currently, no single inflammatory marker provides sufficient discrimination to justify the transition to everyday clinical use as a prognosticator in HF. However, comparisons with well-established markers such as NT-proBNP and other prognostic tools such as cardiac ${ }^{123}$ I-mIBG imaging using receiver operating characteristics (ROCs) and HF risk models are mandatory.

In this issue of the journal, Verschure et $\mathrm{al}^{5}$ analyzed the relationship of the severity of HF with cardiac sympathetic innervation derangement (assessed with ${ }^{123}$ I-mIBG scintigraphy) and with different serum markers of inflammation. They also evaluated the predictive value of cardiac sympathetic innervation derangement and inflammatory markers for cardiac events including ventricular arrhythmia, progression of $\mathrm{HF}$, and cardiac death. The study included 55 patients with moderate stable chronic HF (NYHA class II-III, with no myocardial infarction, hospitalization, or progression of $\mathrm{HF}$ ) on guideline-recommended medical treatment, who were candidates to receive primary implantable cardioverter defibrillator (ICD). All patients underwent cardiac ${ }^{123}$ I-mIBG scintigraphy at $15 \mathrm{~min}$ utes (early) and 4 hours (late) of tracer administration, and early H/M ratio, late H/M ratio, and washout (WO) were calculated. In addition, NT-proBNP and several inflammatory markers including $\mathrm{C}$-reactive protein (CRP), IL-1 $\beta$, IL-6, IL-8, IL-10, IL-12p40, tumor necrosis factor- $\alpha$ (TNF- $\alpha)$, soluble (s)E-selectin, myeloperoxidase (MPO), plasminogen activator inhibitor-1 (PAI-1), tPA, tumor necrosis factor receptor (TNFR) 1 and 2, and interferon (IFN) $\alpha$ and $\beta$ were measured in blood plasma samples, taken just before ${ }^{123}$ I-mIBG administration. Late H/M ratio was significantly correlated with LVEF and NT-proBNP. Additionally, WO was significantly correlated with LVEF, NYHA and NT-proBNP. The only significant independent predictors of ${ }^{123} \mathrm{I}-\mathrm{mIBG}$ parameters on multivariate regression analysis were LVEF for the late $\mathrm{H} / \mathrm{M}$ ratio and NT-proBNP for the WO. During a median follow-up of 34 months ( 2 to 58 months), 13 patients $(24 \%)$ experienced cardiac events (ventricular arrhythmia, $\mathrm{N}=4$; progression of $\mathrm{HF}, \mathrm{N}=4$; and cardiac death, $\mathrm{N}=5$ ). Comparison between patients with and without cardiac events showed no significant differences in late $\mathrm{H} / \mathrm{M}$ ratio and WO. However, patients with events had higher levels of NT-proBNP and CRP, and lower levels of MPO as compared to patients without events. Univariate Cox regression analysis showed that the risk of a cardiac event was associated with CRP (HR 1.047 [1.013-1.081]), NT-proBNP (HR 1.141 [1.011-1.288]), MPO (HR 0.998 [0.996-1.000]), and late H/M ratio (HR 0.182 [0.035-0.946]). Nevertheless, multivariate Cox regression analysis showed that only CRP, NT-proBNP, MPO, and IL-12p40 were the predictors of a cardiac event.

These preliminary results in a small sample of patients with stable HF seem to indicate that cardiac sympathetic activity and inflammation are not related to each other, which may be true, theoretically, attending to the dual activation of the chronic inflammation that occurs in HF (dependently and independently of neurohormonal activation).

However, these results are in contrast to several former reports both in patients with $\mathrm{HF}$ and idiopathic dilated cardiomyopathy (IDCM), ${ }^{6-9}$ and patients without $\mathrm{HF}$ but with chronic inflammation (parainflammation), ${ }^{10}$ showing reduced cardiac sympathetic innervation associated with elevated levels of inflammatory cytokines.

Parthenakis et al's ${ }^{6}$ study included 47 patients with IDCM, NYHA class II-III, and LVEF $30.6 \%$ and found that late H/M ratio was significantly correlated with IL1, TNF, and sTNFRII. Moreover, multivariate linear regression analysis revealed that late $\mathrm{H} / \mathrm{M}$ ratio was independently associated with IL-1 levels. Similarly, Turpeinen et $\mathrm{al}^{7}{ }^{7}$ in a study of 99 patients with IDCM and LVEF $40 \%$, reported that late $\mathrm{H} / \mathrm{M}$ ratio and $\mathrm{WO}$ significantly correlated with IL-6 and NT-proBNP. In addition, those patients who had an adverse cardiac event $(20 \%)$ including death, heart transplantation, or application of biventricular pacemaker or ICD at a follow-up (median 4.1 years) had a higher WO and lower late $\mathrm{H} / \mathrm{M}$ ratio than patients without an event. Messias et $\mathrm{al}^{8}$ recently reported that WO was significantly correlated with IL $1 \beta$ levels, and thus demonstrate that an excessive sympathetic activity influences the systemic inflammatory response. Shinohara et $\mathrm{al}^{9}$ reported that cardiac sympathetic dysfunction and systemic inflammation improved in HF patients who responded to cardiac resynchronization therapy (CRT). Furthermore, the reduction in systemic inflammation was associated 
with the improvement in cardiac sympathetic dysfunction. They evaluated 27 patients with chronic HF of nonischemic origin who underwent CRT. There were 9 responders and 8 non-responders according to echocardiographic findings (15\% absolute decrease in LV endsystolic volume). Only the responders showed a significant increase of the late mean $\mathrm{H} / \mathrm{M}$ ratio and a significant decrease of serum levels of high-sensitive CRP (hs-CRP). Stepwise multiple regression analysis showed that the reduction in hs-CRP level was independently associated with the increase in the late $\mathrm{H} / \mathrm{M}$ ratio.

Diakakis et al, ${ }^{10}$ addressed the relationship of cardiac sympathetic innervation and circulating levels of proinflammatory cytokines in 22 patients with impaired glucose tolerance (IGT), a condition related with early atherosclerotic process and parainflammation. These patients had lower H/M ratio and higher WO as compared to subjects of the control group. Patients with IGT also showed significantly elevated TNF-a, soluble TNF receptor II (sTNFRII), and IL-6 levels compared to controls. Additionally, IL-6 and sTNFRII were significantly correlated with the WR. Therefore, also in patients without HF but with chronic inflammation status, sympathetic derangement seems to be related to the elevated proinflammatory cytokine levels.

Different patient characteristics and treatment may well explain differences between these studies and the present one of Verschure et $\mathrm{al}^{5}{ }^{5}$ where $60 \%$ of the patients had HF of ischemic origin in front of the nonischemic condition of the patients from the other reports. In addition, mean LVEF of the patients studied by Verschure et $\mathrm{al}^{5}$ was $22.4 \%$, lower than that of the former reports. Along with this, it would have been interesting to know the proportion of patients under statin, aspirin, and mineralocorticoid receptor antagonist treatment, which may affect serum levels of inflammatory mediators, but it is not specified in the study.

The study of Verschure et $\mathrm{al}^{5}$ supports the concept that sympathetic innervation derangement and serum markers of inflammation may reflect different aspects of HF progression. Therefore, both parameters might potentially be used complementarily as prognostic factors in HF. However, discrepancies with the previous published reports warrant further studies with major number of patients before definite conclusions could be extracted.

\section{References}

1. Jacobson AF, Narula J. Introduction to cardiac neuronal imaging: A clinical perspective. J Nucl Med 2015;56:3S-6S.

2. Nakajima K, Nakata T. Cardiac 123I-MIBG imaging for clinical decision making: 22-year experience in Japan. J Nucl Med 2015;56:11S-9S.

3. Jacobson AF, Senior R, Cerqueira MD, Wong ND, Thomas GS, Lopez VA, et al. Myocardial iodine-123 meta-iodobenzylguanidine imaging and cardiac events in heart failure results of the prospective ADMIRE-HF (AdreView Myocardial Imaging for Risk Evaluation in Heart Failure) Study. J Am Coll Cardiol 2010;55:2212-21.

4. Mann DL. Innate immunity and the failing heart: The cytokine hypothesis revisited. Circ Res 2015;116:1254-68.

5. Verschure DO, Lutter R, van Eck-Smit BLF, Somsen GA, Verberne HJ. Myocardial ${ }^{123} \mathrm{I}-\mathrm{mIBG}$ scintigraphy in relation to markers of inflammation and long-term clinical outcome in patients with stable chronic heart failure. J Nucl Cardiol 2016. doi:10.1007/s12350-016-0697-7.

6. Parthenakis FI, Patrianakos A, Prassopoulos V, Papadimitriou E, Nikitovic D, Karkavitsas NS, et al. Relation of cardiac sympathetic innervation to proinflammatory cytokine levels in patients with heart failure secondary to idiopathic dilated cardiomyopathy. Am J Cardiol 2003;91:1190-4.

7. Turpeinen AK, Vanninen E, Magga J, Tuomainen P, Kuusisto J, Sipola P, et al. Cardiac sympathetic activity is associated with inflammation and neurohumoral activation in patients with idiopathic dilated cardiomyopathy. Clin Physiol Funct Imaging 2009;29:414-9.

8. Messias LR, Carreira MA, Miranda SM, Azevedo JC, Benayon $\mathrm{PC}$, Rodrigues RC, et al. Do interleukin-1beta levels correlate with MIBG and exercise parameters in heart failure? Arq Bras Cardiol 2013;100:395-403.

9. Shinohara T, Takahashi N, Saito S, Okada N, Wakisaka O, Yufu $\mathrm{K}$, et al. Effect of cardiac resynchronization therapy on cardiac sympathetic nervous dysfunction and serum C-reactive protein level. Pacing Clin Electrophysiol 2011;34:1225-30.

10. Diakakis GF, Parthenakis FI, Patrianakos AP, Koukouraki SI, Stathaki MI, Karkavitsas NS, et al. Myocardial sympathetic innervation in patients with impaired glucose tolerance: Relationship to subclinical inflammation. Cardiovasc Pathol 2008; 17:172-7. 